\title{
The Dynamic Complexity of Enterprise IT System: An Adaptive Perspective
}

\author{
Bing Bai ${ }^{1}$, Pei-kai Hua ${ }^{2}$, and Zhi-qiong Guo ${ }^{3}$ \\ ${ }^{1)}$ Business School of Jiangsu Normal University, Xuzhou, Jiangsu, China \\ ${ }^{2)}$ Business School, Jiangsu Normal University, Xuzhou, Jiangsu, China \\ ${ }^{3}$ Business School, Jiangsu Normal University, Xuzhou, Jiangsu, China
}

\begin{abstract}
Under the tide of information economy, information technology has become the basic conditions for enterprise survival and development. The environment of IT system is becoming more and more complex, and this needs objectively to establish adaptive IT system to promote the formation of IT capability and gain the competitive advantage. This paper analyzes the dynamic complexity of enterprise IT system and its influence on the enterprise performance from adaptive perspective, and tries to reveal the internal complex mechanism of IT system.
\end{abstract}

Keywords - dynamic complexity, IT system, adaptive

\section{企业 IT 系统的动态复杂性：适应性的视角}

\section{白冰 华培凯 郭志琼}

江苏师范大学商学院，徐州，江苏，中国

摘 要 在信息经济的浪潮下, 信息技术已经成为企业赖以生存的基本条件。企业 IT 系统的环境变的越来越复杂, 这就需要企业构 建适应性的 IT 系统来促进 IT 能力的形成, 进而获得竞争优势。本文从适应性的视角, 分析了企业 IT 系统的动态复杂性及其对企业绩 效的影响，试图揭示 IT 系统内部的复杂性机制。

关键词 动态复杂性, IT 系统, 适应性

\section{1. 引言}

在信息经济的浪潮下，信息技术（information technology, IT）对企业的影响极其深远。从外部环境看, 如同 18 世纪中叶发生的那场工业革命, 信息革命对原有经 济、社会、文化各个方面都带来了前所未有的冲击。这无 疑加剧了环境的变化, 使企业所面临的环境变得更加难以 预测, 从而也对企业适应能力提出了更高的要求; 从内部 看, 企业 IT 系统的出现也为企业运作提供了一种强有力的 工具, 企业可以用它来支持决策和改善内部管理、再造业 务流程、创新商业模式等。同时, 企业内外部环境的改变 反过来又会对 IT 系统产生影响。这就客观上要求企业 IT 系统具备适应各种变化的能力, 具有良好的动态适应性。

在动态复杂环境下, IT 系统需要能够灵活应对, 而且
要求 IT 系统能够具有对自身进行改进、扩充和完善的能力。 企业 IT 系统灵活的适应动态复杂环境的变化已经成为企业 获取竞争优势的关键因素, 因此有关适应性 IT 系统的能力 研究应运而生。企业所处环境的动态复杂性是研究 IT 系统 适应能力的最主要原因。企业的环境导致了 IT 系统运行的 不确定性, 只有不断提高 IT 系统适应环境的能力, 才能为 企业构建核心竞争力提供支撑。

\section{2. 适应性概念内涵}

“适应性（adaptive）” 原属于生物学范畴内的术语, 用于解释生物种群的进化与生存环境的关系, 即生物物 种、种群或个体可以通过改善自身状况来更好地适应生存 环境的变化, 以保证种群生存和延续的一种能力。20世纪

国家自然科学基金支持(资助号：71302188)、江苏省高校哲学社会科学基金(资助号：2013SJB6300032) 和 江苏省 “青蓝工程” 资助。 
30年代, Bertalanffy提出一般系统论, 其主要理念在于将 系统与环境之间的相互作用作为理解世界的起点, 划定系 统与环境的动态边界, 研究系统与环境的相互适应, 即关 于适应性的条件、机制、规律的研究成为一般系统论的基 础性内容之一。其后适应性的思想逐渐应用到经济社会、 企业管理以及其他领域中, 不同的学科因研究视角不同, 对适应性的理解也互不相同。1994年霍兰教授提出了复杂 适应系统理论。他认为系统主体是一个基于规则的适应性 主体, 主体内部的规则能够根据环境的变化而改变, 且能 够进行创新。并提出了 “适应性造就复杂性” 的思想, 为 复杂系统的适应性研究提供了新视角, 在管理中得到广泛 应用。从复杂适应系统的角度来看, 适应性是指复杂系统 中的主体在不断的对环境变化做出反应过程中, 通过学习 逐渐调整自身结构以及策略来适应新环境的过程。企业IT 系统是一个复杂系统, 在这个复杂系统里有多个主体 (人) 的相互运动, 而导致了IT系统的复杂适应性特征。适应性 是一个动态的概念, 因为环境在变, 主体需要适应环境才 能生存, 那么它也应该不断地改变。

与IT系统的适应性相关的概念还有柔性 (Flexibility) 和敏捷性 (Agility)。柔性是指主体在处理那些因某种原因 不可控的、不可预测变化的活动时, 以较小的代价进行变 化或反应的能力。敏捷性是主体的一种应激反映, 是对外 界环境变化的快速响应能力。在敏捷性的定义中反应速度 是敏捷性的基本特征, 同时也是敏捷性的关键所在。

从以上的定义可以看出, 适应性强调的是一种主动性 和智能性, 柔性强调是一种灵活性, 而敏捷性强调的是一 种快速性。而从复杂适应系统理论的角度来分析, 适应性 是一个综合性很强的概念, 其内涵中包括了主动适应与学 习、快速、灵活的调整自身的结构和策略等概念, 从复杂 系统的角度来说, 本文认为适应性综合了柔性、敏捷性的 特征。因此, 在对适应性IT系统的研究中, 借鉴了以往对 IT系统柔性、敏捷性的研究成果。

\section{3. 适应性企业与适应性 IT 系统}

（1）适应性企业

将适应性的概念引入企业管理中有学者提出了适应性 企业的概念。适应性企业 (Adaptive Enterprise) 作为一种 管理概念, 最早由S.H.Haeckel于1995年提出, 其标志是在 美国《Planning Review》杂志上发表的一篇题为 “Adaptive Enterprise Design: The Sense-and-Respond Model” 的文章, 并在其随后的著作 《Adaptive Enterprise: Creating and Leading Sense \& Respond Organizations》中对此作了系统、 全面地阐述。适应性企业是信息时代企业运行的一种崭新 的战略思想。其基本思想是面对不可预测的、迅速的市场
环境变化, 企业惟一的战略就是使自己变得更加具有适应 性, 能够对影响企业运行的各种变化因素意识得更及时, 反应得更迅速。适应性企业思想的提出, 受到了学术界的 大量关注, 一些学者分别从不同的角度对其进行了深入地 研究。

Shank (1999) 提出了对组织进行模块化设计, Kusnic 和Owen (1999) 从组织的合作决策分析角度, 分别拓展了 适应性企业战略的核心思想。

Kapoor等（2005）提出了支持适应性企业 “感知一响 应” 模式的一个技术框架, 以帮助企业快速地适应商务环 境变革的要求。

J Ramanathan（2005）基于分形理论, 分析了适应性企 业的结构, 并在此基础上提出了适应性企业 “需求与计 划、执行和交付” 的三阶段运作模式。

张铁男 (2005) 、张亚娟和韩兵 (2009) 结合进化理 论对适应性企业战略进行了分析, 并基于惯例研究提出了 适应性企业战略机制的 “刺激一意识一反应” 模式及其实 现过程。

程雪婷和齐中英 $(2005,2006)$ 基于CAS理论, 对企业 适应性机制的构成要素进行了分析, 认为其主要由管理者 和员工经验、政策及管理制度、组织结构等三类要素构 成, 并研究了我国石油企业的战略与组织结构的适应性演 化问题。并鉴生物学、医学领域的研究成果, 基于组织免 疫的新视角, 探讨企业如何在复杂不确定的环境中通过提 高自身的组织免疫力来增强其适应性的问题。

$\mathrm{Wu}$ 等 (2009) 借助于计算机仿真模型, 对构成适应性 特征的敏捷性、鲁棒性、弹性和抗毁性等四个维度进行了 分析。

可星等 (2013) 以CAS理论中"适应性主体"的概念为 基础, 运用适应性主体的基本模型, 从微观视角来对企业 组织能力系统的适应性进化过程进行分析, 并初步构建了 企业组织能力系统进化的适应性主体基行为模型。

王峰 (2015) 提出无论外部和内部环境如何, 任何一个 企业都试图构建一个属于自己的商业模式来参与竞争。并 分析了企业的商业模式适应性演进的机理

适应性企业的研究总体来说还处在理论探索阶段, 目 前的研究成果还主要在理论层面, 缺乏实践的研究, 尤其 缺乏对适应性企业架构的研究。在信息技术日益发展的今 天, 企业的IT系统越来越成为支撑企业运作的必不可少的 资源和能力, 因此要想建立起适应性的企业, 必须有适应 性的IT系统支持。

(2) 适应性IT系统

将适应性企业的思想引入企业的IT系统建设和规划 中, 本文提出适应性IT系统的概念。根据复杂适应性系统 
理论和适应性企业理论, 本文将适应性IT系统定义为面对 信息技术日益变革的动态复杂环境, 企业IT系统的战略目 标就是使自身变得更加具有适应性, 能够对影响IT系统运 行的各种变化因素迅速反应、及时调整, 进而使得企业IT 系统能够更好的适应环境的变化, 将企业战略发展、业务 以及IT系统做到最佳的融合, 为企业带来IT能力。

\section{4. 企业 IT 系统外部环境的复杂性分析}

适应性是信息时代企业运行的一种崭新的战略思想。 适应性IT系统是一个具有全新架构、能够有效应对不断变 化的内外部环境的IT系统。企业构建适应性IT系统的动因 是来源于企业IT系统面临的内外部环境的动态复杂性。

企业IT系统的外部环境是一个由自然、社会、经济等 系统组成的复杂巨系统。这个复杂的巨系统具有多维度、 多层次、多目标、非线性等特征, 尤其是在经济全球化和 信息技术飞速发展的时代, IT 外部环境的变动越来越广 泛、快速、不确定和复杂。主要表现在以下方面:

(1) 信息技术变革的加剧发展

在当今社会, 信息技术在无时无刻的发生着变革。物 联网、云计算、移动商务和虚拟化等新技术和思想风起云 涌, 使得人们的生活方式和企业的运作模式都在发生着根 本性的变化, 同时也使得企业IT系统面临变革的巨大压 力。企业要想在信息技术日益变革的竞争环境里获得竞争 优势, 必须紧紧把握信息技术发展的趋势, 及时更新自身 的IT系统和技术, 才能适应这种复杂的外部环境。

(2) 经济全球化的影响

世界市场正在融为一体，商品、服务、人才、资金、 信息和技能的跨国自由流动已成为大势所趋。企业面临的 市场不仅仅在是单独国内市场, 而是面临全球的市场范 围, 这加剧了企业所处的环境的复杂性。全球化使得企业 运作不再局限于一个国家, 而且将企业在本国学到的竞争 技能应用于全球, 把全球市场作为利用其竞争优势的广阔 场所, 这导致企业IT系统也面临国际化的竞争。

（3）企业边界日益模糊

信息技术、经济全球化及各种技术的交叉渗透使的传 统产业界线、企业边界日益模糊, 企业与企业之间的联系 越来越密切。很多企业都突破原有的竞争模式、运作模式 及组织边界和产业界限的束缚, 将自己的价值链与其他企 业的价值链结合起来, 形成优势互补, 重新建立企业的竞 争优势。企业边界的日益模糊使得企业所处的竞争环境更 加复杂, 这使得企业对IT系统提出了更高的要求。

(4) 宏观政策的多变

近年来, 全球经济和金融环境复杂多变, 国家根据全 球及国内经济环境不断进行调整宏观政策。同时, 国家根
据全球及国内产业发展形势, 不断调整产业政策。这些政 策的调整对企业发展前景和发展环境带来更多的压力和挑 战。而作为支撑企业运行的IT系统也必然会受到这些政策 的影响。企业IT部门要根据这些政策变化的影响, 及时调 整IT战略, 适应企业战略变化的需求。

上述因素使得企业IT面临的外部环境变得更加动态复 杂, 当然, 一方面外部环境本身确实在发生着剧烈的变 化, 另一方面, 由于企业IT系统逐步对外部环境的适应, 也加剧了外部环境的复杂性, 即霍兰教授所说的 “适应性 造就复杂性”。

\section{5. 企业 IT 系统内部环境的复杂性分析}

企业系统是一个复杂适应系统, IT系统作为企业系统 的子系统, 同样是一个复杂适应系统, 所面临的内部环境 具有复杂性的特征。表现为如下方面:

(1) 内部各系统之间关系的复杂性

企业内部环境各子系统、各要素之间联系广泛而紧 密, 构成一个复杂的关系网络。企业IT系统面临的内部环 境涉及企业研发、生产、营销等其他部门和系统。IT系统 是支撑这些系统和部门正常运转的支持系统, 如何有效的 发挥IT系统的支撑力量以及协调好各个系统之间的关系是 非常复杂的。同时, IT系统本身也具有多个子系统, 这些 子系统的有效配合是保证IT系统正常的前提, 正确的配置 这些IT资源是一项复杂的工程。

(2) 系统结构的复杂性

IT系统结构包括IT的软件体系和硬件体系以及技术架 构等多个方面, 这些IT系统的要素种类数量多, 具有不同 的层次。其中硬件体系是最基础的层次, 而技术架构和软 件体系位于硬件体系之上, 这些系统之间具有很强的耦合 作用。高度耦合使得整个IT系统的结构复杂性增强。

（3）内部运作的智能性

企业IT系统内部环境是有人的参与的复杂适应性系 统, 它具有自学习、自适应、自组织的特性和能力, 能够 在发展过程中不断学习, 不断重组与完善自身的层次结构 与功能结构。IT内部人员能够根据外部市场环境的变化, 及时调整IT系统的架构和配置, 表现为一种智能性, 而这 种智能性表现为企业IT系统对环境的适应性, 而适应性势 必会造成复杂性。

(4) 系统演化的复杂性

企业IT内部环境是动态变化的, 能够根据外部环境的 变化进行调整和适应, 这种调整和适应使得IT系统在不断 的向前发展和演化。但是, 演化的路径具有一定的不确定 性和随机性, 经常会出现分岔和突变的现象, 这使得整个 系统演化过程具有动态复杂性。 


\section{6. 结论}

企业 IT 系统所面临的内外部环境均具有动态复杂性, 客观上要求企业 IT 系统具备一定的适应性, 能够根据环境 变化的需要, 不断的调整 IT 系统的配置, 使 IT 系统的运行 更加顺畅。而适应性 IT 系统正是符合了这一要求。适应性 IT 系统能够对影响 IT 系统运行的各种变化因素迅速反应、 及时调整, 进而使得企业 IT 系统能够更好的适应环境的变 化, 将企业战略发展、业务以及 IT 系统做到最佳的融合, 这是企业构建适应性 IT 系统的动因。企业一旦建立起具有 适应性的 IT 系统, 将会使得的 IT 系统对企业业务流程和战 略发展的支撑力加强, 为企业在激烈的市场竞争中占据一 席之地提供技术保障。

\section{参考文献(References)}

[1] Correal H. LLinking Extendibility, Uncertainty and Variability in Manufacturing Systems. England: Avebury Publishing,1994.

[2] Kidd P. T. Agile Manufacturing: Forging a New Frontier. Addison-Wesley Publishing Company, 1994.

[3] Haeckel $\mathrm{S}$ H. Adaptive Enterprise Design: the Sense-and-Respond Model. Planning Review, 1995, 23(3):6-13.

[4] Haeckel S H. The Adaptive Enterprise: Creating and Leading Sense \& Respond Organizations. Harvard Business School Press, 1999.

[5] Shank M. Creating the Modular Organization. In: Haeckel S H.
The Adaptive Enterprise: Creating and Leading Sense \& Respond Organizations. Harvard Business School Press, 1999.

[6] Kusnic M., Owen D. Collaborative Decision-making in Adaptive Enterprises. In: Haeckel S H. The Adaptive Enterprise: Creating and Leading Sense \& Respond Organizations. Harvard Business School Press, 1999.

[7] Kapoor S., Bhattacharya K., Buckley S., et al. A Technical Framework for Sense-and- Respond Business Management. IBM Systems Journal, 2005, 44(1):5-24.

[8] Ramanathan J. Fractal architecture for the adaptive complex enterprise. Communications of the ACM, 2005, 48(5):51-57.

[9] Zhang Tienan. Research on Adaptive Enterprises Stratagem based on Evolution Theory. Harbin Engineering University, 2005, 2005.5.

[10] Cheng Xueting. Study on the Adaptability Mechanism of Oil Enterprises on the basis of CAS Theory. Harbin Institute of Technology,2006.

[11] Wu J., Hu B., Zhang Y. An Agent-based Simulation Study for Exploring Organizational Adaptation. Simulation, 2009, 85(6):397-413.

[12] Robertson D.A. Agent-Based Models of a Banking Network as an Example of a Turbulent Environment: the Deliberate vs. Emergent Strategy Debate Revisited. Emergence: A Journal of Complexity Issues in Organizations and Management, 2003, 5(2):56-71. 